\title{
Profile of Intelligent Hybrid Information System in Mobile World
}

\author{
Xin $\mathrm{Qi}^{1,2} \cdot$ Weina Fu ${ }^{1,2} \cdot$ Shuai Liu ${ }^{1,2}$ \\ Accepted: 24 December 2021 / Published online: 24 January 2022 \\ (c) The Author(s), under exclusive licence to Springer Science+Business Media, LLC, part of Springer Nature 2022
}

\section{Editorial:}

Mobile application has a very important strategic position in the current world, and mobile Information processing has been acted as an important research domain for a long time [1]. In long-distance transportation, the mobile traffic control system is high depended on effective and timely information transmission [2]; and the intelligent monitoring system is also required more effective anomaly detection [3]. In general mobile world, which includes industrial, education, and other environment, there are also many information-based problems need to be focus on $[4,5]$. Therefore, this issue aims to provide an opportunity for researchers to publish their gifted theoretical and technological studies of intelligent information processing in various mobile networks, as well as their novel applications within this domain. Finally, this issue received 41 submissions and accepted 11 out of them with at least 2 rounds of strict reviews, with acceptance ratio $26.83 \%$. This editorial is divided into two sections, which aims to the intelligent information processing in mobile Ad-hoc networks in the first section, and mobile information processing for real world application in the second section.

A Local Betweenness Centrality Based Forwarding Technique for Social Opportunistic IoT Networks Ritu Nigam, Deepak Kumar Sharma, Satbir Jain, Gautam Srivastava

Design of a Conflict Prediction Algorithm for Industrial Robot Automatic Cooperation Kaiyong Li, Xiaochun Cheng

AGEN-AODV: An Intelligent Energy-Aware Routing Protocol for Heterogeneous Mobile Ad-hoc Networks Mohammad Nabati, Mohsen Maadani, Mohammad Ali Pourmina

Intelligent Spacing Selection Model under Energy-saving Constraints for the Selection of Communication Nodes in the Internet of Things Jing-shu Sun, Teng Zhu, Marcin Woźniak

Mobile Networks-on-Chip Mapping Algorithms for Optimization of Latency and Energy Consumption Arvind Kumar, Vivek Kumar Sehgal, Gaurav Dhiman, S. Vimal, Ashutosh Sharma, Sangoh Park

Image Feature Location Method based on Improved Wavelet and Variable Threshold Segmentation in Internet of Things. Jianhu Gong, Mu-Yen Chen

\section{Intelligent Information Processing in Mobile Ad hoc Networks}

The first section of this issue includes six papers, which focuses on the novel intelligent information processing methods in mobile Ad hoc networks, such as opportunistic IoT Networks, Industrial Robot and classifications [6-11].

The first paper titled "A Local Betweenness Centrality Based Forwarding Technique for Social Opportunistic IoT Networks", authored by Gautam Srivastava from Brandon University, Canada, proposed a novel forwarding scheme named "A Local Betweenness Centrality based Forwarding Technique" (LBCFT) for Social Opportunistic IoT Networks. The scheme used a reduction strategy to discard the inefficient devices which are existed in searching for the right relay node from a massive number of devices due to

A Delay-tolerant Data Congestion Avoidance Algorithm for Enterprise Cloud System based on Modular Computing, Kaiyin Yuan, Feng Wang, Zbigniew Marszalek

Real-time Distribution Algorithm for Fully Comparison Data based on Storm, Chang-qing Dong, Chen Chen, Nver Ren, Jianjun Cai

Design of Mobile Intelligent Evaluation Algorithm in Physical Education Teaching, De-kun Jiang, Fida Hussain Memon

Evaluation of Multimedia Learning Resource Classification Retrieval based on Decision Tree Hashing Algorithm, Yin-zhen Zhong, Wu-xue Jiang

Virtual Reconstruction System of Building Spatial Structure Based on Laser 3D Scanning under Multivariate Big Data Fusion, Yuan Liang, Marcin Woźniak

Shuai Liu

liushuai@hunnu.edu.cn

Weina Fu

fuwn@hunnu.edu.cn

1 Hunan Provincial Key Laboratory of Intelligent Computing and Language Information Processing, Hunan Normal University, Changsha, China

2 College of Information Science and Engineering, Hunan Normal University, Changsha, China 
the growing heterogeneous devices in the networks. A new centrality measurement called local betweenness centrality is proposed to form the significant overlapping communities of network devices to boost forwarding. The performance of LBCFT is evaluated through ONE Simulator. Simulation results showed effectiveness of the proposed LBCFT by compared with traditional protocols, such as Supernode, GRAD, BubbleRap, and PROPHET.

Industrial robot is an important application of mobile technology. The second paper titled "Design of a Conflict Prediction Algorithm for Industrial Robot Automatic Cooperation" authored by Xiaochun Cheng from Middlesex University, United Kingdom, designed a conflict prediction algorithm for the cooperation of industrial robots. The proposed algorithm aimed to solve communication conflicts in traditional industrial robot cooperation which was generated because of the lack unified standards for industrial robot signal to automatic predict. A fuzzy rule for conflict prediction is designed by taking chaos characteristics before and after the collision as feature descriptor. A conflict prediction tool (QPME) is used to compare the distribution of conflict types and the prediction of different conflict rates associated with different recovery strategies. Experimental results show that the proposed algorithm can quantitatively analyze the impact of both hardware and software conflicts on robot cooperation performance effectively.

The third paper titled "AGEN-AODV: An Intelligent Energy-Aware Routing Protocol for Heterogeneous Mobile Ad-hoc Networks", authored by Mohsen Maadani from Islamic Azad University, Iran, proposed a novel heterogeneous Mobile Ad-hoc Network (MANET) routing protocol called "learning Automata and Genetic based Ad hoc OnDemand Distance Vector" (AGEN-AODV), in which routes are rated based on energy, stability, traffic, and hop-count criteria. The proposed protocol focused on MANET consisted of mobile nodes with limited energy resources, and used Genetic Algorithm (GA) in conjunction with Learning Automata (LA) to select the optimal route in order to provide a generalized solution for routing optimization. The LA ran concurrent to the GA to prevent the GA from divergence or sub-optimal convergence. Compared with recent related works, both the throughput, packet delivery ratio (PDR), delay, network lifetime, and energy consumption had obvious advantages.

Current IoT communication node spacing selection process show may potential areas for improvements such as high delay ratio, high total energy consumption ratio, confusion of the optimal communication information band, intelligent spacing node design under the constraints of the energy-saving selection of IoT communication. In this way, the forth paper titled "Intelligent Spacing Selection Model under Energy-saving Constraints for the Selection of Communication Nodes in the Internet of Things", authored by Marcin Woźniak from Silesian University of Technology, Poland, proposed a solution under energy-saving constraints. The interference nodes under the intrusion of the Internet of Things were identified to prevent the generation of serious noisy nodes and frequency hopping data. The simulation results show that the established model not only reduces energy consumption of nodes, shortens the average transmission delay of nodes, but also improves anti-interference effect of node spacing selection.

The fifth paper titled "Mobile Networks-on-Chip Mapping Algorithms for Optimization of Latency and Energy Consumption", authored by Sangoh Park from Chung-Ang University, South Korea, proposed a series of mapping algorithms, Horological Mapping (HorMAP), Rotational Mapping (RtMAP) and Divide and Conquer Mapping (DACMAP) for mapping of tasks onto topology having different cores, minimizing the latency, queuing time, service time and energy consumption of topology. HorMAP algorithm visited the cores one by one guaranteeing load balancing over the grid. RtMAP assigned the task to the cores in rotation one by one guaranteeing the least latency involved during mapping of tasks. DACMAP provided an assurity of load balancing on the grid. Experimental results showed the comparison of proposed algorithms with traditional random mapping algorithm, which 2D mesh topology with $\mathrm{XY}$ routing was considered for the simulation of proposed algorithms.

In Internet of things environment, intelligent visual image often had high noise. Current location method was hard to locate the target when the transition "interference" between the segmented targets was too high or the brightness difference between targets was too large. Aiming at this problem, the sixth paper titled "Image Feature Location Method based on Improved Wavelet and Variable Threshold Segmentation in Internet of Things", authored by Mu-Yen Chen from National Cheng Kung University, Taiwan, proposed a feature location method for variable threshold segmentation image based on improved wavelet. The experimental results showed that even under severe occlusion and high noise conditions in the IoT environment, the proposed method had better image feature location and denoising performance than traditional methods too. The proposed method effectively improved the peak signal-to-noise ratio and denoising accuracy, and shortened the running time. 


\section{Mobile Information Processing in Real Application}

Then, the second section of this issue includes the other five papers, which focuses on the mobile information processing in real application, such as enterprise, automobile, education, as well as Architectural Engineering [12-16].

The seventh paper titled "A Delay-tolerant Data Congestion Avoidance Algorithm for Enterprise Cloud System based on Modular Computing", authored by Zbigniew Marszalek from Silesian University of Technology, Poland, designed a delay tolerant data congestion avoidance algorithm based on modular computing to solve the large delay in traditional large-scale enterprise cloud system. The resource allocation rules of RBFR strategy in traditional enterprise cloud were improved to change the traditional interactive overall mode. The design of delay tolerant data congestion avoidance algorithm is realized by using modular constructing the data congestion control algorithm, mainly in the control layer, through the introduction of ant colony algorithm in modularization. Comparative experiments were designed to verify the algorithm's congestion avoidance effect, and the results showed that the packet loss rate of large enterprise cloud system with the proposed algorithm is lower than traditional algorithms.

Current data allocation algorithms neglect the problems of unsatisfactory allocation results and long execution time caused by the redundancy of full comparative data and the complexity of data types. To solve these problems, the eighth paper titled "Real-time Distribution Algorithm for Fully Comparison Data based on Storm", authored by Chang-qing Dong from China Automotive Technology and Research Center (CATARC) Software Testing (Tianjin) Co.,Ltd, China, as well as Tianjin University, proposed a real-time allocation algorithm of full comparison data based on Storm. The phase unwrapping algorithm of minimum spanning tree was used to remove redundant data in full comparison data; and then the distributed data clustering algorithm and storm framework were used to cluster the full comparison data. Compared with the traditional algorithms, the proposed algorithm had the lowest execution time, the highest acceleration ratio and the closest allocation cost to the ideal overhead.

The ninth paper titled "Design of Mobile Intelligent Evaluation Algorithm in Physical Education Teaching", authored by Fida Hussain Memon from JEJU National University, South Korea, designed and applied a new mobile intelligent algorithm for the teaching evaluation of mobile physical education. The proposed algorithm is used for the calculation error and the long time consuming of the traditional algorithm. In the proposed method, an intelligent BiLSTM model intelligent annotated the sequential sports tasks. The weight of evaluation index was determined and the quantitative evaluation model was established. In experiment, the accuracy results showed that the proposed algorithm had higher evaluation accuracy than the traditional algorithm, the fluctuation range of the evaluation results indicated that the proposed algorithm has good stability, and the application time verified that the proposed algorithm had short time consuming performance.

Current classification and retrieval methods are affected by the amount of data in the classification of multimedia learning resources, and there are problems such as low classification accuracy, low retrieval rate, and long retrieval time. To solve this problem, the tenth paper titled "Evaluation of Multimedia Learning Resource Classification Retrieval based on Decision Tree Hashing Algorithm", authored by Wu-xue Jiang from the Education University of Hong Kong, Hong Kong, proposed a new multimedia learning method. Decision tree collected and classified multimedia learning resources, hash algorithm preprocessed the resources, and the Lyapunov theorem obtained features. Two different deep convolutional networks were used as non-linear hash functions. Annotated propagation algorithm was used for multimedia classification and resources retrieval. Experimental results showed that the proposed method effectively improved the retrieval accuracy and efficiency than traditional methods.

The eleventh paper titled "Virtual Reconstruction System of Building Spatial Structure Based on Laser 3D Scanning under Multivariate Big Data Fusion", authored by Yuan Liang from Shijiazhuang University of Applied Technology, China, designed a virtual reconstruction system of building space structure with laser 3D scanning technology under the fusion of big multimodal data. The proposed method was used to solve disadvantages of traditional systems such as slow running speed, long time-consuming, and poor rendering effect in virtual reconstruction of architectural spatial structure. The system was equipped with a 3D laser scanner, and the noise interference was reduced by image preprocessing module to complete the hardware design. Experimental results showed that the proposed system had faster speed, more consistent reconstruction color, which indicated that reconstruction results of building space structure were conducive to in-depth study of building space.

Acknowledgements The guest editors are thankful to our reviewers for their effort in reviewing the manuscripts. We also thank the Editin-Chief, Dr. Imrich Chlamtac for his supportive guidance during the entire process. The work is sponsored by Natural Science Foundation of Hunan Province with No.2020JJ4434 and 2020JJ5368; Key Scientific Research Projects of Department of Education of Hunan Province with No.19A312; Key Research Project on Degree and Graduate Education Reform of Hunan Province withNo.2020JGZD025;National Social Science Foundation of China with No.AEA200013; Industry-Academic Cooperation Foundation of the Ministry of Education of China with No.HKEDU-CK-20200413-129. 


\section{References}

1. Shuai W, Xinyu L, Shuai L, et al. 2021 Human Short-Long Term Cognitive Memory Mechanism for Visual Monitoring in IoTAssisted Smart Cities. IEEE Internet of Things Journal, online first, https://doi.org/10.1109/JIOT.2021.3077600

2. Liu S, Wang S, Liu X, et al. 2021 Human Memory Update Strategy: A Multi-Layer Template Update Mechanism for Remote Visual Monitoring, IEEE Transactions on Multimedia, online first, https://doi.org/10.1109/TMM.2021.3065580

3. Shuai L, Xinyu L, Shuai W et al (2021) Fuzzy-Aided Solution for Out-of-View Challenge in Visual Tracking under IoT Assisted Complex Environment. Neural Comput Appl 33(4):1055-1065

4. Liu S, Guo C, Al-Turjman F et al (2020) Reliability of response region: A novel mechanism in visual tracking by edge computing for IIoT environments Mech. Syst. Signal Process 138:106537

5. Gao P, Li J, Liu S (2021) An Introduction to Key Technology in Artificial Intelligence and big Data Driven e-Learning and e-Education. Mobile Networks and Applications 26(5):2123-2126

6. Ritu N, Deepak K S, Satbir J, et al. 2021 A Local Betweenness Centrality Based Forwarding Technique for Social Opportunistic IoT Networks, Mobile Networks and Applications, online first, doi: https://doi.org/10.1007/s11036-021-01820-7

7. Kaiyong L, Xiaochun C. 2021 Design of a Conflict Prediction Algorithm for Industrial Robot Automatic Cooperation, Mobile Networks and Applications, online first, doi: https://doi.org/10. 1007/s11036-021-01819-0

8. Mohammad N, Mohsen M, Mohammad A P. AGEN-AODV: An Intelligent Energy-Aware Routing Protocol for Heterogeneous Mobile Ad-hoc Networks, Mobile Networks and Applications, 2021, online first, doi: https://doi.org/10.1007/ s11036-021-01821-6

9. Jing-shu Sun, Teng Zhu, Marcin Woźniak. 2021 Intelligent Spacing Selection Model under Energy-saving Constraints for the Selection of Communication Nodes in the Internet of Things, Mobile Networks and Applications, online first, doi: https://doi. org/10.1007/s11036-021-01828-z

10. Arvind K, Vivek K S, Gaurav D, et al. Mobile Networks-on-Chip Mapping Algorithms for Optimization of Latency and Energy Consumption, Mobile Networks and Applications, 2021, online first, doi: https://doi.org/10.1007/s11036-021-01827-0

11. Jian-hu G, Mu-Yen C. 2021 Image Feature Location Method based on Improved Wavelet and Variable Threshold Segmentation in Internet of Things, Mobile Networks and Applications, online first, doi: https://doi.org/10.1007/s11036-021-01822-5

12. Kaiyin Y, Feng W, Zbigniew M. 2021 A Delay-tolerant Data Congestion Avoidance Algorithm for Enterprise Cloud System based on Modular Computing, Mobile Networks and Applications, online first, doi: https://doi.org/10.1007/s11036-021-01826-1

13. Chang-qing D, Chen C, Nver R, et al. 2021 Real-time Distribution Algorithm for Fully Comparison Data based on Storm, Mobile Networks and Applications, online first, doi: https://doi.org/10. 1007/s11036-021-01824-3

14. De-kun J, Fida H M. 2021 Design of Mobile Intelligent Evaluation Algorithm in Physical Education Teaching, Mobile Networks and Applications, online first, doi: https://doi.org/10.1007/ s11036-021-01818-1

15. Yin-zhen Z, Wu-xue J. 2021 Evaluation of Multimedia Learning Resource Classification Retrieval based on Decision Tree Hashing Algorithm, Mobile Networks and Applications, online first, doi: https://doi.org/10.1007/s11036-021-01823-4

16. Yuan Liang, Marcin Woźniak. 2021 Virtual Reconstruction System of Building Spatial Structure Based on Laser 3D Scanning under Multivariate Big Data Fusion, Mobile Networks and Applications, online first, doi: https://doi.org/10.1007/s11036-021-01825-2
Publisher's note Springer Nature remains neutral with regard to jurisdictional claims in published maps and institutional affiliations.

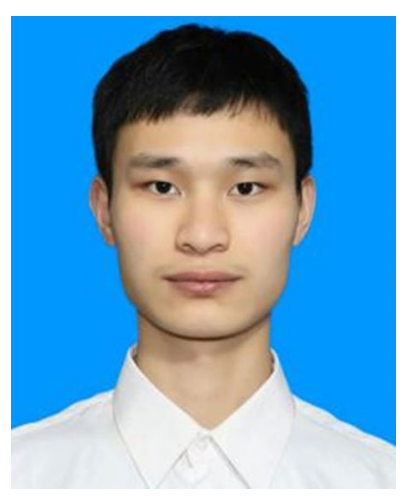

Xin Qi received his B.A. in Computer Science and Technology from Hubei University of Arts and Science in 2021, and now a M. S. student in College of Information Science and Technology, Hunan Normal University. His research interests include artificial intelligence and computer vision.

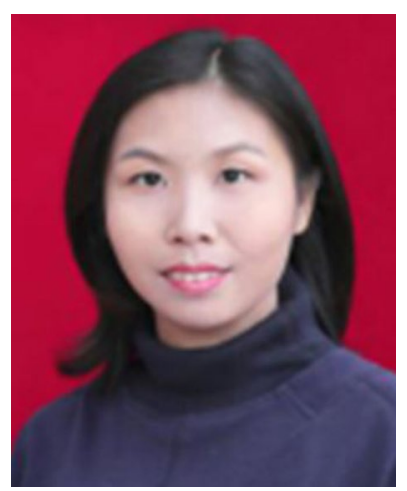

Weina Fu got her BSc from Jilin University, China in 2003, MSc from Jilin University, China in 2006, and PhD from Inner Mongolia University in 2012. Her main research domain is computer vision and video tracking. Now, she is a lecturer in Hunan Normal University, and she has published more than 10 papers.

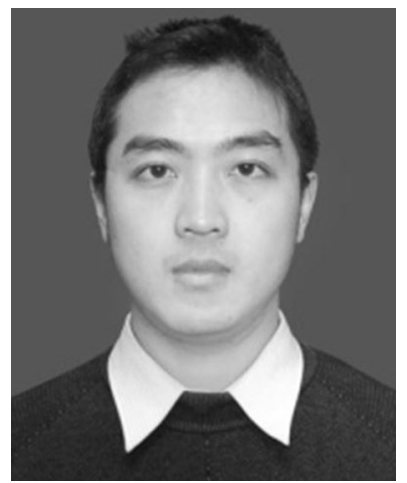

Shuai Liu received his BS, MS and $\mathrm{PhD}$ e from Jilin University in 2002, 2004, and 2011. He used to work as a full professor in College of Computer Science, Inner Mongolia University. Now he acts as a full professor in College of Information Science and Technology, and Vice director of Hunan Provincial Key Laboratory of Intelligent Computing and Language Information Processing, Hunan Normal University $\mathrm{He}$ is now a senior member of China Computer Federation. His research area contains big data processing, computer vision, and information processing. He has published more than 60 papers, with more than 3000 citations. He serves editor role for many respected journals. 